Jeremy J. Ramsden

\title{
Less is different
}

ABSTRACT. Nanotechnology is considered as embodying the qualitative change that occurs when the quantitative change (in this case of dinimishing size) becomes great enough, pace Hegel. Given, however, that although nanotechnology is the "technology of the small" it does not exactly map onto the quantum realm, the domain of the absolutely small. Nanotechnology endeavours to retain most of the quality, specificity and individuality of quantum objects without the state of nano-objects being destroyed by an act of measurement.

Nanotechnology Perceptions 6 (2010) 57-60

Nonsubscribers: purchase individual article 